\title{
Correction to: Application of a Bayesian Watershed Model Linking Multivariate Statistical Analysis to Support Watershed-Scale Nitrogen Management in China
}

\author{
Jian Sha ${ }^{1} \cdot$ Zeli Li $^{1} \cdot$ Dennis P. Swaney ${ }^{2} \cdot$ Bongghi Hong ${ }^{2} \cdot$ Wei Wang $^{3} \cdot$ Yuqiu Wang $^{1}$ \\ Published online: 12 September 2020 \\ (C) Springer Nature B.V. 2020
}

\section{Correction to: Water Resour Manag \\ https://doi.org/10.1007/s11269-014-0696-x}

Unfortunately in the original version of this article, the Electronic Supplementary Material was unintentionally omitted during the publishing process.

Publisher's Note Springer Nature remains neutral with regard to jurisdictional claims in published maps and institutional affiliations.

The online version of the original article can be found at https://doi.org/10.1007/s11269-014-0696-x

Electronic supplementary material The online version of this article (https://doi.org/10.1007/s11269-02002649-7) contains supplementary material, which is available to authorized users.

\author{
Yuqiu Wang \\ yqwang@nankai.edu.cn \\ Jian Sha \\ shajian2004@qq.com \\ Zeli Li \\ zeli.li@qq.com \\ Dennis P. Swaney \\ dps1@ cornell.edu \\ Bongghi Hong \\ bh43@cornell.edu \\ Wei Wang \\ 50850761@qq.com
}

Extended author information available on the last page of the article 


\section{Affiliations}

Jian Sha ${ }^{1} \cdot{\text { Zeli } \mathrm{Li}^{1} \cdot \text { Dennis P. Swaney }}^{2} \cdot$ Bongghi Hong $^{2} \cdot$ Wei Wang $^{3} \cdot$ Yuqiu Wang $^{1}$

1 MOE Key Laboratory of Pollution Processes and Environmental Criteria, College of Environmental Science and Engineering, Nankai University, Tianjin 300071, China

2 Department of Ecology \& Evolutionary Biology, Corson Hall, Cornell University, Tower Road, Ithaca, NY 14853, USA

3 Tianjin Meteorological Science Research Institute, Tianjin 300074, China 